Retrospective Study

\title{
Thoracic Epidural Blood Patches in the Treatment of Spontaneous Intracranial Hypotension: A Retrospective Case Series
}

Paolo Feltracco, MD, Helmut Galligioni, MD, Stefania Barbieri, MD, and Carlo Ori, MD

From: Department of Medicine UO Anaesthesia and Intensive, Care, Padua University Hospital Padua, Italy

Address Correspondence: Paolo Feltracco, MD Department of Medicine UO Anaesthesia and Intensive Care Padua University Hospital, Via Cesare Battisti, 256, 35128 Padua, Italy. E-mail: paolofeltracco@inwind.it

Disclaimer: There was no external funding in the preparation of this manuscript. Conflict of interest: Each author certifies that he or she, or a member of his or her immediate family, has no commercial association (i.e., consultancies, stock ownership, equity interest, patent/licensing arrangements, etc.) that might pose a conflict of interest in connection with the submitted manuscript.

Manuscript received: 02-10-2015 Revised manuscript received: 03-09-2015 Accepted for publication: 03-16-2015

Free full manuscript: www.painphysicianjournal.com
Background: Spontaneous intracranial hypotension $(\mathrm{SIH})$ results from leaks developing in the dura mater. The major symptom is orthostatic headache which gradually disappears after lying down. Lumbar epidural blood patches (EBPs) can be effective in relieving headaches, however, thoracic and cervical EBPs have also been applied to alleviate the symptoms.

Objective and Methods: Retrospective collection of the main characteristics of $\mathrm{SIH}$, site and amount of blood injection, and clinical outcomes of 18 patients who underwent thoracic EBPs for intractable $\mathrm{SIH}$. \{

Study Design: Retrospective case series

Results: All thoracic autologous EBPs except 3 were performed in the sitting position. Patients undergoing epidural puncture at lower thoracic levels (T10-T12) received $25 \mathrm{~mL}$ of autologous blood, $15 \mathrm{~mL}$ and $18 \mathrm{~mL}$ were injected at spinal segments T5-T7 (mid-thoracic) and T2-T4 (upperthoracic), respectively. Thoracic EBPs did not lead to immediate resolution of symptoms in 3 of 18 patients; one of them underwent early repetition with complete headache relief, one refused a second EBP, and one experienced partial resolution, followed by a recurrence, and then satisfactory improvement with a second high thoracic EBP. In long-term follow-up only 2 patients complained of symptoms or relapses.

Limitations: Retrospective nature of the case series, single center experience.

Conclusions: Performing thoracic-targeted EBPs as the preferred approach theoretically improves results with respect to those observed with lumbar EBPs. The immediate response was comparable with that of other reports, but the long-term success rate (90\%) turned out to be very effective in terms of both quality of headache relief and very low incidence of recurrence.

Key words: Central pain, cerebrospinal fluid leak, headache disorders, low intracranial pressure syndrome, epidural blood patch, occipital headache

Pain Physician 2015; 18:343-348

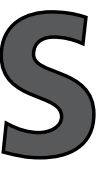
pontaneous intracranial hypotension $(\mathrm{SIH})$ is a quite rare disease in which a leak of cerebrospinal fluid (CSF) through the dura mater causes low CSF pressure within the nervous system and results in a constant string of headaches. Connective tissue disorders affecting the spinal dura have been identified as important causes of SIH (1).

Headaches originating from spontaneous CSF leaks are often associated with tinnitus, visual disturbances, vertigo, and nausea. Symptoms usually occur when the patient is upright and almost always gradually disappear after lying down. Non-severe SIH can be treated with generous amounts of fluids, caffeine, and long rest in a supine position. More serious conditions benefit from an epidural blood patch (EBP), which can be effective either after one procedure or following repeated injections in different epidural spaces.

EBPs have commonly been performed at lumbar 
levels, however, as most spinal CSF leaks occur in the upper segments of the spinal dura, thoracic and cervical EBPs have also been carried out to accelerate the alleviation of symptoms.

Here we briefly report our case series of patients who have been treated with EBPs at various thoracic segments due to intractable $\mathrm{SIH}$.

\section{Methods}

After receiving approval from the local ethics committee and obtaining patients' written informed consent, we retrospectively collected the main characteristics of $\mathrm{SIH}$, demographic data, sites and amount of blood injection, and main clinical outcomes of patients diagnosed as having $\mathrm{SIH}$ who received epidural autologous blood injections at thoracic levels in our institution.

All thoracic autologous EBPs were performed by the same 3 anesthesiologists in the operating room. Autologous blood was collected by another anesthesiologist and injected slowly until the predetermined volume was achieved. Access to the epidural space was made with a 17-gauge Tuohy needle using the lossof-resistance technique with saline. All EBPs except 3 were performed in the sitting position. In 16 out of 21 procedures the median approach was successful, while the paramedian approach was necessary in 5 cases, due to technical difficulties.

After blood injection, patients were tilted downward in the Trendelemburg position for 20 minutes. On completion of the procedure they were sent to the neurological ward with instructions to lie flat for 24 hours, and to limit physical activity in the following days. Discharge occurred only after satisfactory resolution of symptoms, or upon a patient's decision in the case of incomplete remission. Following hospital discharge they were contacted by telephone within the first month, and then at 2 month-intervals for 8 months.

\section{Results}

The main symptom which required EBP was the occurrence of persistent frontal and occipital headaches upon assuming an upright position, associated in $>50 \%$ of cases with a variable intensity of neck stiffness, nausea/vomiting, vertigo, and tinnitus.

Results on demographic characteristics, type of headache, and the most common brain magnetic resonance imaging (MRI) neuroradiological findings at the time of anesthesiological evaluation are summarized in Table 1.

The duration of clinical symptoms before the first EBP ranged from 5 days to 23 weeks, with a mean pretreatment time of 16 days. All patients underwent EBP after initial conservative therapies failed to provide relief from symptoms. Six patients had already received one EBP at lumbar level, 2 underwent their first thoracic EBPs following 2 previous failed lumbar treatments, and one after 3 failed lumbar EBPs (all lumbar EBPs performed in other institutions). In 3 of 18 patients, due to unbearable headache while sitting, thoracic epidural

Table 1. Demographic data ( $M=$ male; $F=$ female), type of headache, and brain MRI findings at the time of anesthesiological evaluation.

\begin{tabular}{|c|c|c|}
\hline $\begin{array}{l}\text { Demographics } \\
\text { Patients } \mathrm{n}=18\end{array}$ & $\begin{array}{c}\text { Gender } \mathbf{F}(\mathbf{n}=\mathbf{1 4}) \\
\text { mean age } 32 \text { yrs (range } 26 \\
-58)\end{array}$ & $\begin{array}{c}\text { Gender } M(\mathbf{n}=\mathbf{4}) \\
\text { mean age } 31 \text { yrs (range } \\
42-66)\end{array}$ \\
\hline \multicolumn{3}{|l|}{ Main Characteristics of Reported Headache } \\
\hline Acute "thunderclap" intolerable soon after assuming the sitting or standing position & 2 & 1 \\
\hline Early onset on standing, but tolerable for a while & 4 & 2 \\
\hline Moderate on standing but debilitating on exertion & 3 & 1 \\
\hline Subacute, gradual onset tension-type chronic headache & 2 & \\
\hline Slight headache, even while lying down & 2 & \\
\hline Worsening headache associated with somnolence and dysarthria. & 1 & \\
\hline \multicolumn{3}{|l|}{ Brain MRI Findings } \\
\hline More or less diffuse pachymeningeal enhancement & 14 & 4 \\
\hline Thin subdural fluid collections & 2 & 1 \\
\hline Downward descent (sagging) of the brain & 1 & 1 \\
\hline Right-hemisphere subdural haematoma with signs of recent bleeding & & 1 \\
\hline
\end{tabular}


punctures and injections were carried out in the lateral decubitus position. The sites of execution and the clinical manifestations during and following autologous blood injections are listed in Table 2.

We have reported the presumed interspaces of execution after anatomical inspection and spinous process palpation had allowed us to approximate the site of puncture. However, as the anatomic landmark-based approach to identify spinal levels is not accurate, the levels of injections should be defined more appropriately as lower, middle, and upper thoracic.

Patients undergoing epidural puncture at lower thoracic levels received $25 \mathrm{~mL}$ of autologous blood, while $15 \mathrm{~mL}$ and 18 $\mathrm{mL}$ were injected at middle (T5-T7) and upper (T2-T4) thoracic segments, respectively.

The degree of headache relief and long-term outcomes are reported in Table 3.

In 3 out of 18 patients, thoracic EBPs did not result in immediate resolution of symptoms. Due to early recurrence of severe headaches, one of these 3 patients underwent repetition of EBP 5 days after the first injection, 2 interspaces above the previous injection site; one patient with moderate headaches refused a second EBP, and within days experienced spontaneous resolution with conservative measures; one had noticeable early improvement but later experienced a recurrence of pain upon intense daily activity only. Eight months later this last patient underwent another EBP carried out in a different way: Through a Tuohy needle at T2-T3 interspace an epidural catheter was pushed cranially toward the cervical segments and $6 \mathrm{~mL}$ of autologous blood were initially injected, then the catheter was removed and additional $16 \mathrm{~mL}$ of blood were subsequently injected through the same needle. Satisfactory improvement was obtained with this procedure, although it did not completely abolish the symptoms.

Besides one patient in whom, due to the early occurrence of significant back pain, only $9 \mathrm{~mL}$ of autologous blood were injected at mid-thoracic interspace (instead of the $15 \mathrm{~mL}$ that had been drawn), no limitations with regard to the injected volume were encountered when the predetermined collected blood was administered at selected thoracic levels.

Major adverse events, blood taps, or signs of radicular damage were not noticed during epidural punctures.

\section{Discussion}

Evidence from the literature demonstrates that moderate or severe forms of $\mathrm{SIH}$, with scarce tendency to be self-limiting, usually benefit from an EBP (2). In our case series, thoracic EBPs were very effective in terms of both degree of headache relief and of minimal incidence of recurrence. Only one of 18 patients required an early second thoracic EBP after a failed previous thoracic injection. Thoracic EBP was also repeated in 2 other patients, due to headache

Table 2. Sites of execution of autologous blood injections (first and repeated injections), and clinical manifestations during and following EBP (24-hour time period).

\begin{tabular}{|l|c|}
\hline \multicolumn{1}{|c|}{$\begin{array}{c}\text { Interspaces of EBPs Execution } \\
\text { (including repeated EBPs) }\end{array}$} & No of EBPs (21) \\
\hline T10-T12 (lower - thoracic) & 8 \\
\hline T5-T7 (mid - thoracic) & 9 \\
\hline T2-T4 (upper- thoracic) & 2 \\
\hline T2-T3 with associated epidural catheter & 1 \\
\hline Double simultaneous EBP at T5-T6 and T11-T12 & 1 \\
\hline
\end{tabular}

\begin{tabular}{|l|l|l||}
\hline Clinical manifestations during and following EBP & $\begin{array}{c}\mathbf{n}^{\circ} \text { of } \\
\text { patients } \\
\text { F }\end{array}$ & $\begin{array}{c}\mathbf{n}^{\circ} \text { of } \\
\text { patients }\end{array}$ \\
\hline Moderate occipital headache lasting > 12 h & 2 & \\
\hline Aspecific neck pain & 3 & 2 \\
\hline Bilateral transient thoracic "burning"pain & 1 & \\
\hline Back pain, with signs of radiculopathy & 1 & \\
\hline Aspecific signs of acute meningeal irritation & 1 & 1 \\
\hline Thoraco-lumbar back pain associated with right leg pain & 1 & \\
\hline Transient dorsal T5-T8 discomfort with paresthesias & 2 & 1 \\
\hline
\end{tabular}

Table 3. Degree of headache relief following the thoracic EBPs and long-term outcomes.

\begin{tabular}{|l|c||}
\hline \multicolumn{1}{|c||}{ Degree of Pain Relief and Long-term Outcome } & $\begin{array}{c}\text { No of } \\
\text { Patients }\end{array}$ \\
\hline Complete headache relief within 24 hours & 15 \\
\hline Persistence of headache, with similar intensity as before the procedure & 1 \\
\hline Residual moderate headache, with spontaneous resolution within 5 days & 1 \\
\hline Persistent but tolerable headache & 1 \\
\hline Sustained pain relief at 16 to 30 months follow-up & 16 \\
\hline
\end{tabular}


recurrence, 5 and 8 months after the first procedure, respectively.

In recent-onset headaches, a time period of approximately one week of conservative treatment was observed before performing the EBP, while in long lasting $\mathrm{SIH}$, the procedure was carried out early upon patient referral.

Based on our previous experience with the lumbar injections, which resulted in unsatisfactory outcomes and a greater than $20 \%$ recurrence of headache (unpublished data), during the last 3 years we have opted for the thoracic level as the preferred first intervention site in all individuals, avoiding the lumbar injection as the first approach.

The region of prevalent distribution of paraspinal extra-arachnoid CSF collection visualized on MRI determined the preferred spinal level where epidural puncture was performed, in what it could be considered an almost "site-targeted EBP."

Although we targeted the thoracic segments with the first EBP, the optimal site of epidural blood injection has not yet been clearly established, and most reports underline that EBPs at the lumbar level are still widely performed (3). It has been demonstrated that blood may travel from the lumbar segments through many spinal segments up in a cephalad direction, with the extent of spread being related to the injection volume (2). Accordingly, lumbar EBPs below the suspected CSF leak are currently performed $(3,4)$ with overall rates of success ranging from $50 \%$ to $80 \%(5,6)$. Knowing that the majority of CSF leaks are found at the cervical-thoracic junction or along the thoracic spine (2), the injection of $30-40 \mathrm{~mL}$ or more of blood at lumbar levels has been recommended to improve these results. It has been recognized that the use of such large volumes, however, may increase the risk of spinal compression, with consequent back pain or radiculopathy (7).

The technique used in our case series differs from the established lumbar practice; thoracic EBPs from the start aims at reducing the amount of blood injected, and theoretically improving the results commonly observed with lumbar EBPs (6). Unlike Griauzde et al (8) who performed large-volume multisite blood patches (an average of $54 \mathrm{~mL}$ ) through a single-catheter advanced under fluoroscopic guidance from a lumbar epidural access superiorly into the midthoracic region, we directly targeted various levels of the thoracic spine. We believe that a thoracic EBP as the first site of intervention represents a better risk/benefit approach, rather than having to perform a repeat procedure after an unsuccessful lumbar puncture. It has been suggested that performing the injections upward towards the thoracic levels may increase the probability that the clots will better cover the sites of leaks (9).

The volumes injected at different thoracic levels were chosen according to the diverse anatomic width of the epidural space at different regions, and also considering the amounts that have been reportedly administered in previous studies (8). The administration of the predetermined amount of blood according to the chosen thoracic interspaces was associated with a very low incidence of periprocedural neurological complications and patient discomfort (Table 2). Early symptoms severe enough to suspend the injection before reaching the desired volume occurred only in one patient.

Based on our expertise in performing high-thoracic epidural punctures and catheterization, we consider EBPs at variable thoracic segments to be practicable, and, in fact, only minor paraesthesias, and very rarely, have occurred.

In this series, one patient who was on chronic anticoagulant treatment, with recurrence of SIH associated with subdural hematoma, underwent a double EBP at different thoracic segments (T5-T6 and T11-T12) during the same session. Wang et al (10) reported a successful treatment of subdural hematomas SIH-related with targeted cervico-thoracic or lower thoracic EBPs.

Although whether the site of delivery of EBP may result in a clear advantage in terms of effectiveness, and reduced incidence of relapse is still uncertain, our approach turned out to be very effective in the longterm follow-up.

Within the first 48 hours, all except $2 \mathrm{SIH}$-related headaches had disappeared. Disabling postural symptoms post-blood injection still persisted in one young man, but were promptly resolved with a second thoracic EBP.

In this series the immediate response to EBP was comparable with results from other studies, which report a $60-90 \%$ early success rate $(11,12)$; however, the long-term quality of pain relief appears to be remarkably superior. At 2-month-interval phone calls up to $>16$ months, all patients but 2 reported they were completely asymptomatic; no patients required referral to the neurological department, and none of them complained of any symptoms or relapses severe enough to hinder their normal life-style.

Some studies have reported an incidence of complete pain relief approximating $80 \%$ or less at several months follow-up $(12,13)$. Our almost $90 \%$ success rate 
of lasting subjective improvement in symptoms is very similar to the reports by Ferrante et al (14) and Chung et al (15) in which, apart from some differences in procedural approach and time-delay between start of symptoms and treatment, excellent short- and longterm outcomes were described. These findings are also comparable with the percentage of pain relief observed with CT-guided blood patching targeted at observed leak sites (16).

It is postulated that the headache alleviation after an EBP is due to more of a compression effect than actually sealing the leak $(3,5)$. Although lumbar EBPs may temporarily raise the epidural pressure and block the leak, they may lose this ability once the pressure has dissipated. At the thoracic level, this compression effect may be obtained with a relatively low volume of blood, which may easily reach the cervical meninges, extending and distributing to the regions where many CSF leaks occur.

Although most anesthesiologists refrain from considering thoracic EBPs as the first approach, our recent practice aims at performing lower thoracic EBPs as the first-line invasive treatment of $\mathrm{SIH}$, without requiring demonstration of CSF leakage site, and with the possibility of choosing higher interspaces in cases of relapse of symptoms or after previous ineffective lumbar attempts. The "one-time double EBP," via epidural catheter first, and Tuohy needle immediately afterwards, was also attempted to enable access of blood to cover a relatively distant upper site of dural tear. Blood injection into the cervical epidural space via an epidural catheter, with improvement of the headache, has been recently reported by Kwon et al (17) and Wang and Wang (18).

Limitations of this study are the retrospective nature of the case series, the single center experience, the limited number of EBP providers, and the potential limited reproducibility in institutions without expertise in performing high thoracic epidurals.

More data is needed to validate whether the upper or lower thoracic site of injection, the "double EBPs in the same sitting," and the predetermined volumes injected have an essential impact on the effectiveness of the intervention.

\section{Conclusion}

In conclusion, thoracic EBP as the first-line approach proved to be useful and with long-lasting beneficial effects in severe $\mathrm{SIH}$, not recovering with conservative treatment or not improving after a lumbar EBP. In a physician's hands which are accustomed to performing thoracic epidurals on a routine basis, the application of this technique may be considered quite a safe procedure, as its risk approximates the risk of central neuraxial block implemented for routine thoracic/ abdominal surgery.

\section{Conflict of interest statement:}

The authors declare that they have no conflicts of interest.

The authors assert no personal pecuniary in the writing of this article. No writing assistance was utilized in the production of this manuscript.

There is no funding/support for this study.

None of the authors contributing to this manuscript are employees of a company, stockholders, nor consultants for a company, and none are members of a speakers' bureau.

\section{Authors' contributions}

PF designed the study, collected and analyzed the data, drafted the

manuscript, and gave final approval of the manuscript. HG collected the data and gave final approval of the manuscript. SB contributed to acquisition of data, analyzed the data, drafted the manuscript, and gave final approval of the manuscript. CO designed the study, analyzed the data, drafted the manuscript, and gave final approval of the manuscript.

\section{Acknowledgments}

We are grateful to Dr. Annachiara Cagnin and Dr. Patrizia Rossi for their kind support in identifying and investigating $\mathrm{SIH}$ patients and during periprocedural assistance. 


\section{References}

1. Schievink WI. Spontaneous spinal cerebrospinal fluid leaks. Cephalalgia 2008; 28:1345-1356.

2. Schievink WI. Spontaneous spinal cerebrospinal fluid leaks and intracranial hypotension. JAMA 2006; 17:2286-2296.

3. Yoon SH, Chung YS, Yoon BW, Kim JE, Paek SH, Kim DG. Clinical experiences with spontaneous intracranial hypotension: a proposal of a diagnostic approach and treatment. Clin Neurol Neurosurg 2011; 113:373-379.

4. Vakharia SB, Thomas PS, Rosenbaum AE, Wasenko JJ, Fellows DG. Magnetic resonance imaging of cerebrospinal fluid leak and tamponade effect of blood patch in postdural puncture headache. Anesth Analg 1997; 84:585-590.

5. Horikoshi T, Watanabe A, Uchida M, Kinouchi $\mathrm{H}$ Effectiveness of an epidural blood patch for patients with intracranial hypotension syndrome and persistent spinal epidural fluid collection after treatment. Neurosurg 2010; 113:940-946.

6. Hannerz J, Dahlgren G, Irestedt L, Meyerson B, Ericson K. Treatment of idiopathic intracranial hypotension: Cervicothoracic and lumbar blood patch and peroral steroid treatment. Headache 2006; 46:508-511.

7. Coombs DW, Hooper D. Subaracnoid pressure with epidural blood "patch." Reg Anesth 1979; 4:3-6.
8. Griauzde J, Gemmete JJ, Chaudhary N Wilson TJ, Pandey AS. Large-volume blood patch to multiple sites in the epidural space through a single-catheter access site for treatment of spontaneous intracranial hypotension. AJNR 2014; 35:1841-1846.

9. Nipatcharoen P, Tan SG. High thoracic/ cervical epidural blood patch for spontaneous cerebrospinal fluid leak: A new challenge for anesthesiologists. Anesth Analg 2011; 113:1476-1479.

10. Wang J, Zhang D, Gong X, Ding M. Rapid resolution of subdural hematoma after targeted epidural blood patch treatment in patients with spontaneous intracranial hypotension. Chin Med J 2014 127:2063-2066.

11. Berroir S, Loisel B, Ducros A, Boukobza M, Tzourio C, Valade D, Bousser MG. Early epidural blood patch in spontaneous intracranial hypotension. Neurology 2004; 63:1950-1951.

12. Franzini A, Messina G, Nazzi V, Mea E, Leone M, Chiapparini L, Broggi G, Bussone $G$. Spontaneous intracranial hypotension syndrome: A novel speculative physiopathological hypothesis and a novel patch method in a series of 28 consecutive patients. J Neurosurg 2010; 112:300-306.

13. Kong DS, Park K, Nam DH, Lee Jl, Kim
JS, Eoh W, Kim JH. Clinical features and long-term results of spontaneous intracranial hypotension. Neurosurgery 2005; 57:91-96.

14. Ferrante E, Arpino I, Citterio A, Wetzl R, Savino A. Epidural blood patch in Trendelenburg position pre-medicated with acetazolamide to treat spontaneous intracranial hypotension. Eur J Neurol 2010; 17:715-719.

15. Chung SJ, Lee JH, Im JH, Lee MC. Shortand long-term outcomes of spontaneous CSF hypovolemia. Eur J Neurol 2005; 54:63-67.

16. Kranz PG, Gray L, Taylor JN. CT-guided epidural blood patching of directly observed or potential leak sites for the targeted treatment of spontaneous intracranial hypotension. AJNR 2011; 32:832-838.

17. Kwon SY, Kim YS, Han SM. Spontaneous $C_{1-2}$ cerebrospinal fluid leak treated with a targeted cervical epidural blood patch using a cervical epidural Racz catheter. Pain Physician 2014; 17: $E_{3} 81-E_{3} 84$.

18. Wang E, Wang D. Successful treatment of spontaneous intracranial hypotension due to prominent cervical cerebrospinal fluid leak with cervical epidural blood patch. Pain Med 2014 Mar 2512418 [Epub ahead of print] 